\title{
Remifentanil patient controlled analgesia for patients unable to tolerate lithotripsy with enteral analgesia
}

\author{
John Michael Henderson ${ }^{1 *}$, Rosie Jones ${ }^{2}$, Cathy O'Neill' and PVS Kumar ${ }^{1}$ \\ ${ }^{*}$ Correspondence: john_henderson@doctors.net.uk \\ ${ }^{1}$ Harold Hopkins Department of Urology Royal Berkshire Hospital London Road Reading RG1 5AN United Kingdom. \\ ${ }^{2}$ Department of Anaesthetics Royal Berkshire Hospital London Road Reading RG1 5AN United Kingdom.
}

\begin{abstract}
Background: Extracorporeal shock wave lithotripsy is an effective treatment for kidney stones which does not require general anaesthesia and can be performed as a daycase procedure. The fragmentation of calculi can be painful and various analgesic regimens have been described. A small minority of our patients are unable to tolerate ESWL with enteral analgesia. We offered a repeat ESWL treatment with remifentanil Patient Controlled Analgesia (R-PCA) to this group.

Methods: Patients were given oral paracetamol 1g and 100mg rectal diclofenac before ESWL. Remifentanil PCA was prepared to provide a $0.5 \mathrm{mcg} / \mathrm{kg}$ bolus with 2 minute lockout with dose increase as necessary. The primary outcome measure was ESWL energy level tolerated and was compared between groups with the Wilcoxon signed-rank test. Secondary outcomes included pain score, sedation score and adverse effects.

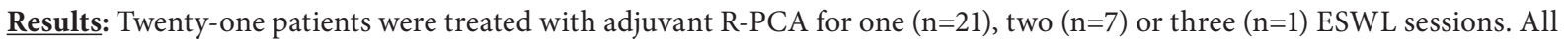
patient were able to tolerate a significantly higher ESWL power level with R-PCA compared to enteral analgesia $(\mathrm{p}<0.01)$. Sedation was minimal (all sedation scores $\geq 3$ ) with a low incidence of side effects.

Conclusion: Patients treated with R-PCA tolerated a significantly higher ESWL power level and all patients completed the treatment. These patients were maintained on their intended treatment modality and avoided the operative and anaesthetic risks of alternative procedures such as ureteroscopy. On demand bolus PCA allows accurate titration of opiate to pain and is safe to use with the limited resources available in the ESWL suite. The combination of this delivery method with the minimal sedation and rapid elimination of remifentanil proved effective and safe with few adverse effects.
\end{abstract}

Keywords: ESWL, remifentanil, analgesia

\section{Background}

The extracorporeal use of shock wave treatment for renal calculi in humans was first described in 1980 [1]. Since then, it has developed into a cornerstone of modern urological practice. Extracorporeal shock wave lithotripsy (ESWL) refers to the targeting of a shockwave onto a calculus to cause fragmentation. The shockwaves are weak, causing minimal damage to tissue through which they are transmitted but then focussed into maximal intensity at the surface of the calculus or focal point (F2). The pain experienced during ESWL is directly proportional to the energy density of the shockwave as it passes through the skin. The high energy density of the early lithotripters such as the Dornier $\mathrm{HM} 3$ required regional or general anaesthesia for the patient. With further advances in equipment and technology, energy levels used were much lower and therefore analgesia requirements decreased [2].

Simple enteral analgesics like diclofenac can provide comparable analgesia to opiates for ESWL. [3] Patients in our centre are given 1 gram of oral paracetamol and 100 milligrams of rectal diclofenac before treatment. They are provided with co-codamol 1 $\mathrm{g} / 16 \mathrm{mg}$ upon discharge. Less than $2 \%$ of our patients given enteral analgesia are unable to complete ESWL treatment due to pain.

The lithotripsy suite is a small area with limited space for complex monitoring equipment. For the patients who are unable to tolerate ESWL with enteral analgesia, an analgesic technique is required which provides effective analgesia with rapid onset and recovery. Patient controlled analgesia (PCA) refers to the delivery of a prescribed quantity of intravenous analgesic upon depression of a control button by the patient. It allows the patient to obtain analgesia without delay, and has safety mechanisms to prevent overdose. Remifentanil (GlaxoSmithKline) is an ultra short-acting synthetic opioid analgesic. It is metabolised by plasma and tissue esterases and therefore accumulation does not occur. The context sensitive half-time, a measurement of drug elimination, for remifentanil is 3.2 minutes compared to 47.3 minutes for alfentanil. [4] It is an ideal drug for short procedures which require potent analgesia with a rapid recovery and early patient discharge. This study evaluated tolerance of ESWL with adjuvant R-PCA in those patients unable to complete treatment with enteral analgesia.

(ㄷ) 2013 Henderson et al; licensee Herbert Publications Ltd. This is an Open access Article distributed under the terms of Creative Commons Attribution License (http://creativecommons.org/licenses/by/3.0). This permits unrestricted use, distribution, and reproduction in any medium, provided the original work is properly cited. 
Henderson et al. Journal of Anesthesiology and Clinical Science 2013,
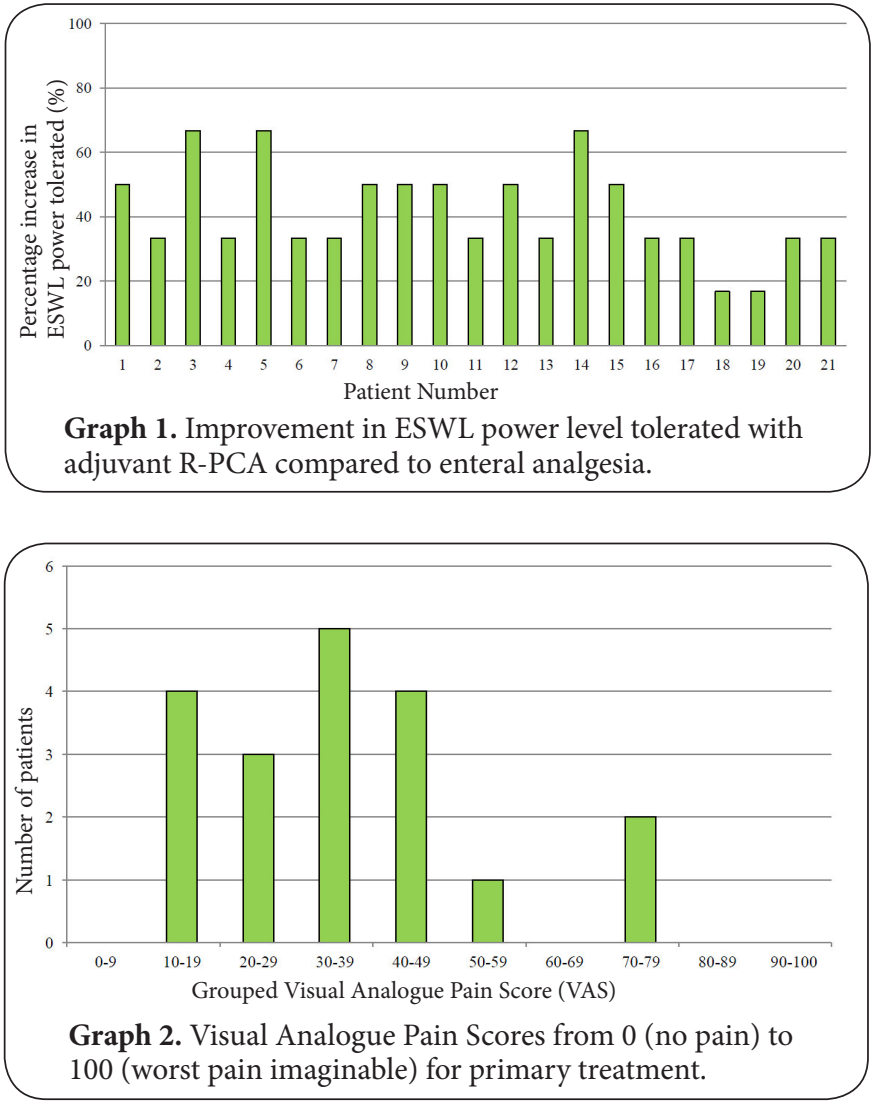

\section{Materials and Methods}

\section{Patients and Exclusion Criteria}

Patient unable to complete ESWL treatment due to pain were offered a repeat treatment with adjuvant R-PCA. Exclusion criteria included patients under the age of 16 , with a contraindication to daycase treatment or history of adverse reaction to opioid analgesia.

\section{Dosage and Treatment}

The study population group were treated on a named patient basis and so did not meet criteria requiring formal ethics committee approval. Patients were consented on an individual basis as to the indications, risks and benefits of the procedure and the off-licence use of remifentanil PCA. Patients were nil by mouth for 6 hours preceding treatment. A $100 \mathrm{mg}$ diclofenac suppository and $1 \mathrm{~g}$ oral paracetamol were given pre-procedure. A consultant anaesthetist prepared remifentanil PCA to provide a $0.5 \mathrm{mcg} / \mathrm{kg}$ bolus with 2 minute lockout. This dose was increased as necessary. Patient sedation was minimised by not administering a background infusion. The anaesthetist monitored the patient throughout treatment. Calculus appropriate ESWL (Stortz Modulith SLX Lithotripter) was administered. Success was defined by the ESWL energy level tolerated. After the treatment, the patient was monitored in recovery and discharged if pain control was adequate and there were no symptoms of nausea or vomiting.

\section{Outcome Measures}

The primary outcome level was ESWL energy level achieved. Secondary outcomes included pain score (Visual Analogue Scale from 1-100), sedation score (1- unresponsive to 5- awake) and adverse effects. If calculus clearance required further ESWL, outcome analysis was repeated separately from the primary R-PCA treatment.

\section{Statistical Analysis}

The ESWL energy level achieved was compared between treatment groups using the Wilcoxon signed-rank test (Graphpad Prism v5, Graphpad Software Inc). Statistical significance was taken as $\mathrm{p}<0.05$.

\section{Results}

\section{Patients and Exclusion Criteria}

Over a two year period, 21 patients were unable to tolerate ESWL treatment due to pain. All accepted the invitation to return for treatment with adjuvant R-PCA. Mean age was 40 years old (range 16-67) with 12 male and 9 female patients. 29 treatment episodes were analysed. Patients were unable to tolerate treatment with enteral ESWL and were treated once $(n=21)$, twice $(n=7)$ or three times $(n=1)$ with adjuvant R-PCA.

\section{Dosage and Treatment}

For primary R-PCA ESWL, the median remifentanil bolus requirement was $0.5 \mathrm{mcg} / \mathrm{kg}$ (range $0.5-0.75 \mathrm{mcg} / \mathrm{kg}$ ). The mean total dosage was $4.4 \mathrm{mcg} / \mathrm{kg}$ (range $2.3-7.9 \mathrm{mcg} / \mathrm{kg}$ ). All patients tolerated the intended treatment of 3200 shocks at energy level 6 . The length of treatment was 25-30 minutes.

\section{Outcome Measures}

All patients were able to tolerate a higher ESWL power level with R-PCA compared to enteral analgesia (Graph 1). The median primary treatment energy level achieved was significantly higher than during prior treatment with enteral analgesia $(p<0.01)$. VAS for primary R-PCA treatment is shown in Graph 2.

\section{Adverse Effects}

Seven patients required supplementary oxygen during the procedure, 1 patient received atropine for bradycardia on second treatment and there were no recorded hypotensive episodes. Sedation was minimal with all patients scoring 3 or above. One patient required admission overnight with pain and vomiting secondary to stone fragmentation and passage.

\section{Discussion}

In this study, we demonstrate that adjuvant R-PCA treatment results in a significant increase in ESWL target energy level tolerated. This effect persists with repeat treatment. Patients who could not tolerate ESWL with enteral analgesia crossed over into the R-PCA treatment group. Although there was no control group, the effect of both analgesic regimens were 
studied in the same population which eliminated group variability. Anaesthetic supervision was mandatory to monitor and treat side effects of treatment.

The mechanism of pain during ESWL is poorly understood. Cavitation of blood and cellular fluid may cause tissue disruption with stimulation of nociceptors. Pain perception is dependent on shockwave factors but also patient pain tolerance. Weber et al., found a range of Threshold of Pain (TP) and Maximal Tolerable Pain (MTP) during ESWL for different patients. $35 \%$ of patients given no analgesia were able to tolerate ESWL. [5] Effective analgesia is important for ESWL to ensure minimisation of patient movement and allow stone targeting and fragmentation. For those patients unable to tolerate ESWL due to pain, options include an alternative stone treatment modality such as ureteroscopy under general anaesthesia, or an alternative analgesic regimen to improve tolerance of ESWL. The minimum effective analgesic concentration (MEAC) is the concentration at which pain effective analgesia is achieved. Intermittent physician administered analgesia may result in multiple troughs below MEAC resulting in pain and poor treatment tolerance. [6] The advantage of PCA is accurate titration of analgesia to patient pain with fewer troughs below MEAC resulting in decreased analgesic requirements compared to physician administered analgesia. [7] The advantage of a PCA opiate such as remifentanil with a short context-sensitive half-time is that of effective analgesia, minimal sedation and rapid recovery. The combination of a proven analgesic delivery system with a fast acting opiate is demonstrated in our study, with all patients able to complete treatment at target energy level and only one patient requiring admission afterwards due to ESWL associated side effects.

The two main dosing methods for PCA are continuous infusion with demand dosing, or demand dosing alone. Continuous infusion with demand dosing may lead to inappropriately high plasma opiate levels with respiratory depression as a background infusion continues irrespective of demand button use. Beloil et al., compared remifentanil and sufentanil during ESWL. No difference in analgesic efficacy, surgeon or patient satisfaction or respiratory depression during ESWL was demonstrated. Patients given remifentanil had less respiratory depression, nausea and vomiting after ESWL than those given sufentanil. [8] It has been shown that a lower basal remifentanil infusion rate decreases the side effects of treatment (nausea, vomiting, itching and dizziness) with no difference in VAS compared to a higher infusion rate. [9] A bolus delivery with no basal infusion dose may lead to a faster recovery with no change in patient comfort or overall satisfaction. [10] In our study, we did not administer a basal infusion and provided a bolus dose, with dose escalation as required. If the patient became sedated, they were unable to depress the demand button and so the sedation wore off without further opiate administration. This negative feedback loop was an important safety feature due to the limited facilities available in the confines of the lithotripsy suite.

This small study was limited to those patients unable to complete their primary treatment modality due to pain. All of these patients then underwent the investigational treatment with no control group. Some of the effect seen may have been due to the enteral and parenteral analgesics working in combination on multiple pain pathways. The disadvantages of R-PCA include the need for supervision at all times by an anaesthetist and the monitoring and delivery equipment required for treatment. The adverse effects of intravenous opiates include nausea, vomiting, sedation and respiratory depression and the recognition and treatment of these is of paramount importance. Previously, the primary alternative treatment for a patient unable to tolerate ESWL would have been ureteroscopy under general anaesthesia. The advantage of an effective analgesic treatment for this small group of patients is that they are maintained on their intended treatment modality without the associated risks of general anaesthesia and operative management.

\section{Conclusions}

The combination of an ultra short-acting opiate like remifentanil with a proven delivery device like PCA has been shown to be effective. The majority of our patients undergoing ESWL tolerate the procedure with simple enteral non-opiate analgesia and we would advocate the continuation of this as a first line treatment. For those unable to tolerate this, R-PCA is a useful adjunct and allowed effective treatment in all cases.

\section{Competing interests}

The authors declare that they have no competing interests.

Authors' contributions

$\mathrm{JMH}, \mathrm{CN}, \mathrm{PVSK}$ and RJ conceived the study, collected and analysed the data. RJ supervised the administration of remifentanil analgesia. PVSK and CN provided the ESWL treatment. JMH drafted the manuscript. All authors read and approved the final manuscript.

\section{Publication history}

Received: 30-Jan-2013 Revised: 11-Feb-2013

Re-Revised: 19-Feb-2013 Accepted: 26-Feb-2013

Published: 05-Mar-2013

\section{References}

1. Chaussy C, Brendel W and Schmiedt E: Extracorporeally induced destruction of kidney stones by shock waves. Lancet 1980, 2:1265-8. I Article | PubMed

2. Chow GK and Streem SB: Extracorporeal lithotripsy. Update on technology. Urol Clin North Am 2000, 27:315-22. | Article I PubMed Abstract | PubMed Full Text

3. Naerger HG: Choosing the correct pain relief for extracorporeal lithotripsy. Br J Urol 1995, 76:145-6. | Article I PubMed

4. Kapila A, Glass PS, Jacobs JR, Muir KT, Hermann DJ, Shiraishi M, Howell $\mathrm{S}$ and Smith RL: Measured context-sensitive half-times of remifentanil and alfentanil. Anesthesiology 1995, 83:968-75. I Article I PubMed

5. Weber A, Koehrmann KU, Denig N, Michel MS and Alken P: What are the parameters for predictive selection of patients requiring anesthesia for extracorporeal shockwave lithotripsy? Eur Urol 1998, 34:85-92. | Article 
Henderson et al. Journal of Anesthesiology and Clinical Science 2013,

http://www.hoajonline.com/journals/pdf/2049-9752-2-18.pdf

PubMed

6. Grass JA: Patient-controlled analgesia. Can Anaesth Soc J 1984, 31:120-

2. | PubMed

7. Kortis HI, Amory DW, Wagner BK, Levin R, Wilson E, Levin A, Pitchford $\mathrm{DE}$ and Pollak $\mathrm{P}$ : Use of patient-controlled analgesia with alfentanil for extracorporeal shock wave lithotripsy. J Clin Anesth 1995, 7:205-10. I Article | PubMed

8. Beloeil H, Corsia G, Coriat P and Riou B: Remifentanil compared with sufentanil during extra-corporeal shock wave lithotripsy with spontaneous ventilation: a double-blind, randomized study. $\mathrm{Br} J$ Anaesth 2002, 89:567-70. | Article | PubMed

9. Medina HJ, Galvin EM, Dirckx M, Banwarie P, Ubben JF, Zijlstra FJ, Klein $\mathrm{J}$ and Verbrugge SJ: Remifentanil as a single drug for extracorporeal shock wave lithotripsy: a comparison of infusion doses in terms of analgesic potency and side effects. Anesth Analg 2005, 101:365-70. | Article | PubMed

10. Sa Rego MM, Inagaki Y and White PF: Remifentanil administration during monitored anesthesia care: are intermittent boluses an effective alternative to a continuous infusion? Anesth Analg 1999, 88:518-22. | Article | PubMed

\section{Citation:}

Henderson J M, Jones R, O’Neill C and Kumar P: Remifentanil patient controlled analgesia for patients unable to tolerate lithotripsy with enteral analgesia. journal of Anesthesiology and Clinical Science 2013, 2:18. http://dx.doi.org/10.7243/2049-9752-2-18 